\title{
Study of electric field in SiPM active volume by the volt-farad characteristics analysis
}

\author{
V. Kushpil ${ }^{1, *}$, V.Ladygin ${ }^{2}$, and S.Kushpil ${ }^{1}$ \\ ${ }^{1}$ Nuclear Physics Institute of the CAS, 25068 Řež, Czech Republic \\ ${ }^{2}$ JINR/LHEP, Dubna, Russia
}

\begin{abstract}
The purpose of this study is to understand the change of the SiPM structure after irradiation. We compared the profile of the electric field in the SiPM (KETEK) active region for ten not-irradiated and irradiated detectors. The standard method of measurement of $\mathrm{C}-\mathrm{V}$ characteristics was applied using two configurations (serial and parallel circuit) to exclude the influence of the serial resistance. Dependencies of capacitance on the frequency were studied in the range from 10 to 1000 $\mathrm{KHz}$. For non-irradiated detectors we detected in CV characteristics the local instability basically connected with accumulation of charge on boundary optical isolation-silicon. In addition, the hysteresis of CV characteristics was detected. For irradiated detectors the local instability was visible as well but the hysteresis of CV characteristics was not detected. The results demonstrate that the applied method can be used for the relative analysis of how SiPM active region properties changed after irradiation.
\end{abstract}

\section{Introduction}

The study of the radiation hardness of SiPM detectors with internal amplification for use in calorimeters in high-energy physics has been carried out during the last decade [1]. In recent years, there has been an interest in the study of the properties of SiPMs operating in neutron radiation fields. These experimental facilities where a fluence of about $10^{13} \mathrm{n} / \mathrm{cm}^{2}$ is expected after several months of operation (experiments CBM [2], NICA [3]). It was previously noted that the changes in the properties of semiconductor detectors depend not only on the intensity of irradiation but also on other factors [4]. It was found that the dark current of the detector which absorbed the fluence of $3 * 10 \mathrm{E}^{8} \mathrm{n} / \mathrm{cm}^{2}$ during the year was less than the dark current of the detector of the same type that was irradiated by $2.5^{*} 10^{12} \mathrm{n} / \mathrm{cm}^{2}$ during 5 hours and undergone the self-annealing for a week. We irradiated two SiPMs of the same KETEK series for several hours until they absorbed the fluence of $\sim 10^{12} \mathrm{n} / \mathrm{cm}^{2}$. We measured the change of the dark current and studied the changes in the internal structure based on the measurement of the capacitance-voltage characteristic [5]. Based on the analysis of capacitance-frequency characteristics we investigated the ratio of the

\footnotetext{
* e-mail: kushpil@ujf.cas.cz
} 
effective concentration of trap centers to the effective lifetime of charge carriers on these traps [6]. This technique allows estimating the change in the spectrum of the detector noise after the irradiation. Then we started tests of detectors of the same series installed in the 10 channel calorimetric module developed and manufactured at JINR [7]. The calorimetric module was irradiated for three years at the neutron source of the U120 cyclotron at the NPI, Řě̌, Czech Republic. The measurements were carried out in the following sequence: several hours of irradiation before reaching the fluence of $\sim 10^{8} \mathrm{n} / \mathrm{cm}^{2}$ and then several months for the self-annealing of defects. In 2017 after the regular irradiation session we found out that the noise increased so much that it was almost impossible to register the light flashes from the scintillator over the background noise. After that, the detectors were removed from the readout system and their studies were carried out in the NPI - ̌̌ež laboratory to study changes in the internal structure, distribution of impurities in the depletion region, changes in the electric field in the detector and changes in the noise spectrum.

\section{Experiment and analysis of measurement results}

The measurement setup, procedure and methods of irradiation were described in our earlier work. The research methodology for current-voltage, voltage-capacitance, and faradfrequency characteristics and measurement setup were presented in [8]. In the data analysis, special attention was paid to the study of errors due to temperature changes and ambiguity in the interpretation of volt-farad characteristics for various measurement circuits (serial vs parallel). Evaluation criteria given in [9] and additional studies carried out in our laboratory allowed us to select the serial measurement scheme. The method used for the calculation of the electric field profile in the detector is based on the data that we obtained for the impurity concentration profile. The analysis of the farad-frequency characteristics was carried out in the standard way described earlier. To confirm our assumptions about the specific changes in the spectral density of the noise after irradiation, we performed a Fourier and Wavelet noise spectrum analysis for not irradiated and irradiated detectors at the same bias voltage and at a temperature of $+25^{\circ} \mathrm{C}$.

\section{Discussion of the results}

As we expected based on previous measurements of KETEK detectors irradiated with neutrons the most important changes for operation of SiPM occur in the near-surface layer. Performing the calculation of the electric field in the depletion region, we can obtain reliable results only at distances deeper than $0.5 \mu \mathrm{m}$ under the surface. In Fig. 1 we see that the depletion region decreases for irradiated detectors. 


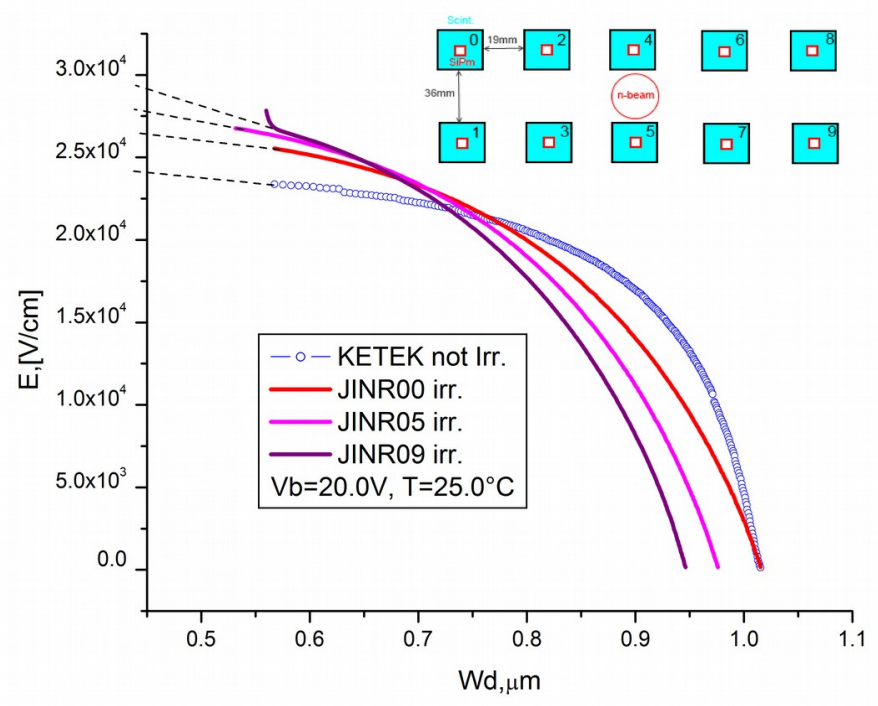

Figure 1. Electric field into SiPM depletion region for irradiated and not irradiated detectors $\left(\mathrm{T}=25^{\circ} \mathrm{C}\right.$, Vbias $\left.=20 \mathrm{~V}\right)$

Fig. 2 demonstrates that the detectors N5 and N6 located near the center of the beam have smaller changes in depletion depths and impurity concentrations in comparison with the detectors N9 and N10 located farther away from the beam. This leads to the conclusion that a neutron beam of $2 \mathrm{~cm}$ diameter does not scatter spherically symmetric on scintillators as we assumed in our previous works.

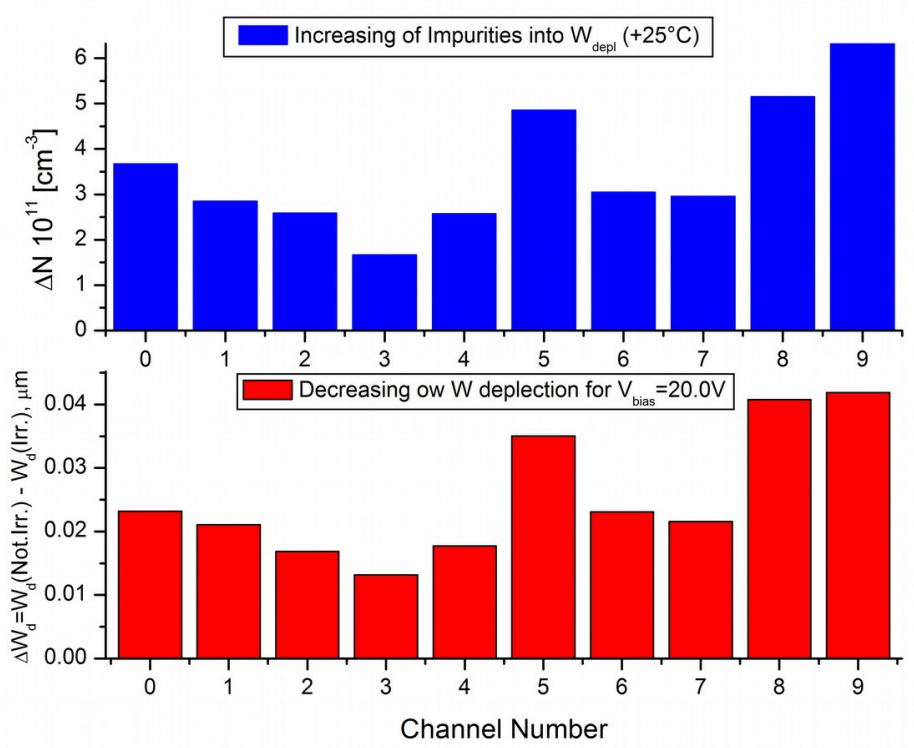

Figure 2. Increasing integral of concentration impurities (1) and decreasing depletion region for SiPM for irradiated detectors 
The most interesting are results obtained by the analysis of farad-frequency characteristics (Fig. 3). KETEK detectors irradiated by a small fluence during the long time scale have a much higher change in the $\langle\mathrm{T}>\mid<\mathrm{N}\rangle$ ratio compared to detectors irradiated shortly by a four orders of magnitude larger fluence. The Fourier analysis method showed that the spectral density of noise increased uniformly across the whole bandwidth after irradiation but no obvious peculiarities were found in the spectral distribution of noise. The wavelet analysis method showed the difference in spectra of irradiated and non-irradiated detectors in more details. However, the region of our interest from $10 \mathrm{KHz}$ to $1000 \mathrm{KHz}$ seems to be too narrow compared with the total $2 \mathrm{GHz}$ band that we studied. This does not allow us to clearly distinguish specific features of the noise spectrum that we expected around $40 \mathrm{KHz}$.

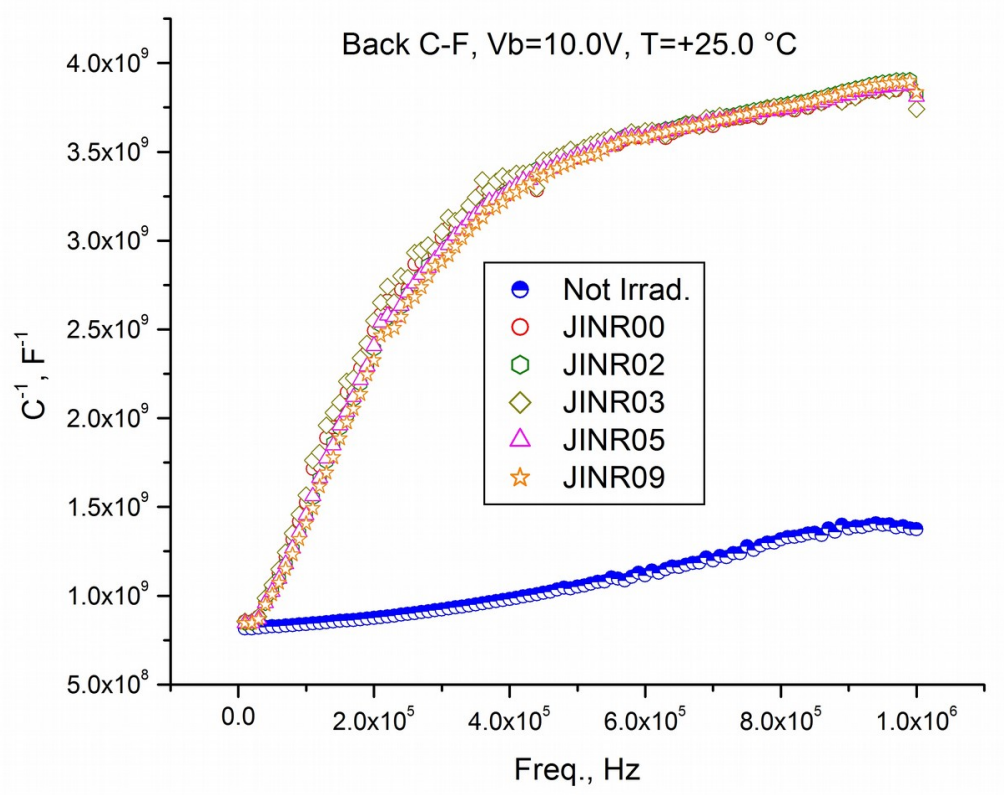

Figure 3. Change of the ratio $<\mathrm{T}>/<\mathrm{N}>$ for irradiated and not irradiated SiPM obtained from the analysis $\mathrm{C}-\mathrm{F}$ characteristics $\left(\mathrm{T}=25^{\circ} \mathrm{C}\right.$, Vbias $\left.=10.0 \mathrm{~V}\right)$

\section{Conclusions}

We showed that the change in the electric field inside the depletion region is due to an increase in the effective impurity concentration and a corresponding decrease in depth of the depletion region. We failed to explore the most interesting region of $0.5 \mu \mathrm{m}$ just under the surface due to the complex internal structure of the studied detectors. We confirmed the assumption that noise characteristics change more drastically for neutron irradiation by the smaller neutron fluence over a long time than by the higher fluence over a short time. The study of changes in the noise density spectrum did not allow us to identify the characteristic differences in the noise spectra for irradiated and non-irradiated detectors. Perhaps, we 
should study the spectra in a narrower frequency range. We can approximate the electric field profile from the examined area of $0.5-1.1 \mu \mathrm{m}$ under the detector surface to the area of $0-0.5 \mu \mathrm{m}$ under the surface (see Fig. 1). The consequent simulation of the charge collection process would show that the corresponding change in the electric field results in an increase in the rising time of the current pulse. Note, that here we consider the voltage operating region below $20 \mathrm{~V}$ where the avalanche amplification does not occur yet. As a result, this will lead to a deterioration of the time resolution in case of the use of this type of SiPM detectors in the TOF system.

The work was supported in part by the JINR-Czech Republic Scientific Cooperation Programs and Plenipotentiary of Czech Republic at JINR grants in 2017-2018. The authors thank the NPI cyclotron and neutron generators team, especially M. Štefánik and M. Majerle, for excellent beam conditions and help with the irradiation test, which was carried out at the CANAM infrastructure.

\section{References}

1. Erika Garutti, JINST 6, C10003 (2011)

2. Claudia Höhne (CBM collaboration), JPCS 420, 012016 (2013)

3. V.D. Kekelidze et al., EPJ Web of Conferences 71, 00127 (2014)

4. F. Hartmann, Radiation Damage in Silicon Detector Devices, in Springer Tracts in Modern Physics, 275 (Springer, 2017)

5. V. Kushpil et al., Nucl.Instrum.Meth. A 787, 117-120 (2015)

6. V. Kushpil et al., JPCS 675, 012039 (2016)

7. V. Kushpil et al., PoS Baldin ISHEPP XXII, 133 (2015). doi:10.22323/1.225.0133

8. V. Mikhaylov et al., PoS EPS HEP 2015, 282 (2015). doi:10.22323/1.234.0282

9. J. Lauwaert et al., Solar Energ. Mater. Solar Cells 94(6), 966-970 (2010) 